\title{
Proposta de sistema de medição de desempenho gerencial para uma empresa de projetos de arquitetura
}

\author{
Proposal for a management performance measurement system for \\ an architectural design firm
}

Propuesta de sistema de medición de desempeño de gestión para una empresa de diseño arquitectónico

\author{
AMARAL, Tatiana Gondim do ${ }^{1}$ \\ SOUZA, Maressa Ramos ${ }^{2}$ \\ 1 Escola de Engenharia Civil e Ambiental, Universidade Federal de Goiás, Goiânia - GO, Brasil. \\ tatiana_amaral@hotmail.com
} ORCID: 0000-0002-9746-4025

2 Escola de Engenharia Civil e Ambiental, Universidade Federal de Goiás, Goiânia - GO, Brasil. maressars@gmail.com ORCID: 0000-0001-5888-0571

Recebido em 01/02/2019 Aceito em 20/02/2020 


\title{
Resumo
}

Ao longo dos anos, diversas mudanças criaram um contexto favorável para a implementação de sistemas de medição de desempenho (SMD) nas organizações, o que conduziu a uma diversidade de pesquisas relacionadas ao assunto. Especificamente na construção civil, uma vasta literatura sobre SMD é encontrada, entretanto, pouco se investiga no âmbito das empresas de arquitetura. Diante do exposto, foi desenvolvida uma pesquisa qualitativa, de caráter exploratório, cujo principal objetivo é a proposição de um SMD para uma empresa de projetos de arquitetura. Para o levantamento de dados, foi aplicado um questionário e uma entrevista semiestruturada. Posteriormente, foi proposto um SMD para a empresa. As categorias adotadas para os indicadores foram: stakeholders, eficiência, qualidade, inovação e sustentabilidade, e resultado, que proporcionaram uma estrutura aplicável tanto para o aprimoramento da gestão da empresa estudada, quanto para a implementação em outras empresas.

Palavras-Chave: Sistema de medição; Medição de desempenho; Indicadores; Monitoramento; Empresa de projetos.

\begin{abstract}
Over the years, several changes have created a favorable context for the implementation of performance measurement systems (SMD) in organizations, which has led to a research diversity related to the topic. Moreover, in the construction sphere, a vast literature on SMD has been found, however, within the architectural firms' scopes, just a few have been explored. Therefore, a qualitative exploratory research has been developed, whose main objective is to propose an SMD for an architectural firm. Furthermore, for this case study and data collection, a questionnaire and a semistructured interview have been applied. Subsequently, the SMD have been proposed to the company. Indeed, the categories which have been adopted as indicators were stakeholders, efficiency, quality, innovation and sustainability, and the result, which have provided a framework applicable to both, the management improvement of the studied enterprise and also to the implementation in other organizations.
\end{abstract}

Key-Words: Measuring system; Measuring performance; Indicators; Monitoring; Project firm

\section{Resumen}

Con los años, varios cambios han creado un contexto favorable para la implementación de sistema de medición del desempeño en las organizaciones, lo que ha llevado a una diversidad de investigaciones relacionadas con el tema. Específicamente en la construcción civil, se encuentra una vasta literatura sobre sistemas de medición del desempeño, sin embargo, poco se investiga en el ámbito de las empresas de arquitectura. En vista de lo anterior, se desarrolló una investigación cualitativa de naturaleza exploratoria, cuyo objetivo principal es la propuesta de un sistema de medición del desempeño para una empresa de diseño arquitectónico. Para la recolección de datos, se aplicó un cuestionario y una entrevista semiestructurada. Posteriormente, se propuso un sistema de medición del desempeño para la empresa. Las categorías adoptadas para los indicadores fueron: partes interesadas, eficiencia, calidad, innovación y sostenibilidad, y resultados, que proporcionaron una estructura aplicable tanto para mejorar la gestión de la empresa estudiada, como para su implementación en otras empresas.

Palabras clave: sistema de medición; medición de desempeño; indicadores; monitoreo; empresa del proyecto 


\section{Introdução}

Ao longo dos anos, diversas mudanças criaram um contexto favorável para a implementação de sistemas de medição de desempenho nas organizações, como a evolução do ambiente competitivo, a necessidade de aumentar o número de negócios, a evolução do conceito de qualidade, maior foco na melhoria contínua e desenvolvimento em tecnologia da informação (GARENGO et al., 2005).

Esse cenário tem incentivado pesquisas relacionadas ao desempenho em diferentes campos do conhecimento, como contabilidade, operações, estratégia, recursos humanos e marketing, em vários níveis de análises (FRANCO-SANTOS et al., 2012; DENG et al., 2012).

Não diferente, o tema tem sido amplamente explorado no âmbito da construção civil, um setor em crescente e constante mudança, no qual vêm sendo disponibilizada uma quantidade e acesso rápido à informação, requerendo das organizações uma grande capacidade de adaptação e aprendizagem (MARTINS et al., 2014).

Assim, novas abordagens conceituais surgiram e a medição de desempenho deixou de possuir apenas uma perspectiva estática e unidimensional, em que o objetivo era se concentrar em indicadores de resultado em termos financeiros, e adquiriu uma perspectiva multidimensional (FRANCO-SANTOS et al., 2012). O que levou ao surgimento de um novo desafio para pesquisadores e para a construção civil, medir aspectos qualitativos ou intangíveis de desempenho (JIN et al., 2013), tanto em nível organizacional quanto operacional.

Em suma, medição de desempenho é um processo lógico que se inicia com a necessidade de medir, passa pela seleção do indicador, define os padrões para checagem, compara os resultados alcançados com os respectivos padrões, chega às conclusões e, por fim, atinge a etapa da tomada de decisão. Desse modo, as organizações necessitam estar examinando constantemente o seu desempenho e analisando se ele está de acordo com os objetivos estipulados (SOUZA, 2014).

Embora a indústria da construção conte com inúmeras iniciativas de melhorias, inclusive com o uso de indicadores de desempenho, a literatura ainda aponta que seu desempenho é baixo em comparação com outras indústrias, pela natureza complexa dos projetos, a existência de muitas disciplinas e partes interessadas, processos fragmentados no projeto e a singularidade de cada um (HORSTMAN, 2013).

Segundo Horstman (2013), essas características revelam que o uso de indicadores de desempenho em projetos de construção precisa estar sujeito a alterações ao longo de várias fases e possuir determinada especificidade. Com efeito, isso pode tornar os indicadores menos aplicáveis para acompanhamento em longo prazo, e mais adequados para a natureza única dos projetos.

Além das questões abordadas, a necessidade de mensurar o desempenho é ainda maior pelo fato do crescimento do nível de complexidade nos projetos de edificações. De acordo com Nóbrega Júnior e Melhado (2013), tal complexidade se deve às novas demandas e exigências, como da Norma de Desempenho de Edificações NBR 15575:2013, de sustentabilidade na construção e inserção de ferramentas Building Information Modeling (BIM).

Em vista disso, ressalta-se que há uma vasta literatura sobre medição de desempenho no que tange aos principais conceitos atribuídos, métodos de classificação e implementação. Contudo, os estudos se destinam às médias e grandes empresas, como construtoras, que se divergem das empresas de projetos de arquitetura que são micro e pequenas em sua maioria. De acordo com Madrigal (2012), empresas desse porte possuem um tipo de gestão específica e consideravelmente distinta dos grandes grupos empresariais.

Nesse contexto, observa-se a carência de pesquisas voltadas às empresas de projeto, identificando-se assim uma lacuna de conhecimento a ser preenchida. Dessa forma, o principal objetivo do presente 
estudo é a proposição de um sistema de medição de desempenho gerencial para uma empresa de projetos de arquitetura, com o intuito de contribuir para avanços no gerenciamento de empresas do setor da construção.

\section{Revisão Bibliográfica}

A revisão bibliográfica foi dividida em duas etapas, a primeira com a explanação de alguns conceitos e referências importantes acerca do desempenho, e a segunda com trabalhos recentes selecionados da literatura nacional e internacional, voltados à medição de desempenho na construção civil e no processo de projetos, destacando a contribuição de cada um.

\subsection{Desempenho: conceitos e definições}

Desempenho é um conceito multidimensional e dinâmico, que compreende tanto a esfera do resultado quanto a comportamental, de modo que a ênfase somente na ação é insuficiente. Dessa forma, para o estudo do desempenho, três perspectivas podem ser abordadas e integradas: as diferenças de desempenho entre indivíduos, as questões situacionais que podem estimular ou prejudicar o desempenho, e o ponto de vista do processo com foco no desenvolvimento, no qual a melhoria do próprio processo de ação melhora o desempenho (SONNENTAG e FRESE, 2002). Para Vernadat $(1996)^{1}$ apud Souza (2014), o desempenho é tido como um tríptico: organização, competência e motivação.

Assim como diferentes perspectivas são atribuídas, o desempenho também é analisado em diferentes níveis, com diversas nomenclaturas, como sistema de avaliação de desempenho (SAD) e sua evolução para o sistema de gestão de desempenho (SGD) (RODRIGUES et al., 2017), indicadores de desempenho (BERNARDI, 2013), sistemas de medição de desempenho contemporâneos (FRANCOSANTOS et al., 2012), além das abordagens com foco específico, como indicadores de resultado para projetos (ORIHUELA et al., 2016), indicadores de inovação (PLENTZ, 2014 e BEUREN et al., 2014), dentre outros.

Tendo em vista tal abrangência sobre o assunto, destacam-se as definições feitas por Neely et al. (2005). Segundo os autores, as ações relacionadas ao desempenho que uma organização pode desenvolver, são diferenciadas em medida de desempenho, medição de desempenho e sistema de medição de desempenho, respectivamente uma métrica, um processo e um conjunto de métricas, todos em função de quantificar a eficiência ou eficácia das ações. Convém salientar que os termos métrica, medida e indicador terão o mesmo significado neste estudo, sendo o último mais utilizado a fim de se obter uma uniformidade.

Segundo Fernandes (2004), indicador é o agente tradutor da informação, que democratiza o acesso às informações por todos os interessados, de maneira única e universal. Os indicadores constituem um instrumento fundamental para auxiliar na identificação, acompanhamento e avaliação de fatos de qualquer natureza, permitindo planejar diretrizes para atuação (SOUZA, 2014). Também possibilitam uma avaliação quantitativa do desempenho de qualquer processo, atividade, produto ou serviço, na qual deve haver um padrão de referência esperado para comparações (ORIHUELA et al., 2016).

Todavia, segundo Souza (2014), os indicadores só fazem sentido se forem medidos e analisados em conjunto, o que resulta numa visão holística de um determinado processo ou atividade. Consequentemente, é imprescindível identificar os processos, os parâmetros para o monitoramento e os indicadores mais adequados para gerar informações. Nesse sentido, o sistema de medição de desempenho cumpre a função de agregar e sistematizar os indicadores e ainda nortear o processo de medição.

\footnotetext{
${ }^{1}$ VERNADAT, F. B. Enterprise modeling and integration: principles and applications. London: Chapman \& Hall, 1996.
} 
A medição de desempenho pode exercer vários papéis na dinâmica gerencial. Uma das aplicações mais comuns é a de controle, no entanto, pode assumir um papel fundamental para a alta administração responsável pela tomada de decisões estratégicas e operações em geral (JIN et al., 2013).

Contudo, a razão mais importante para medir o desempenho é apoiar a melhoria, com objetivo de crescimento constante, pois, os sistemas de medição permitem monitorar e impulsionar as organizações para o sucesso, bem como podem minimizar ou eliminar as variações existentes para assegurar a continuidade dos resultados desejados (CELLI, 2014).

\subsubsection{Classificações e critérios de seleção dos indicadores}

Diversas são as formas de classificação dos indicadores de desempenho encontradas na literatura, sendo que existem distintas denominações. De acordo com Costa (2003), os indicadores de desempenho podem estar agregados de diferentes maneiras, conforme as necessidades de informação da empresa e sua estrutura de organização e decisão. Algumas destas classificações podem ser observadas na Tabela 1.

Tabela 1: Classificação de Indicadores.

\begin{tabular}{|c|c|c|}
\hline Autor & Classificação & Descrição \\
\hline \multirow[t]{2}{*}{ Lantelme (1994) } & $\begin{array}{l}\text { Indicadores de } \\
\text { desempenho específicos }\end{array}$ & $\begin{array}{l}\text { Fornecem informações para o gerenciamento da empresa e } \\
\text { de seus processos individuais. }\end{array}$ \\
\hline & $\begin{array}{l}\text { Indicadores de } \\
\text { desempenho global }\end{array}$ & $\begin{array}{l}\text { São utilizados para avaliar o desempenho do setor como um } \\
\text { todo e podem ser voltados à empresa ou podem ser } \\
\text { setoriais. }\end{array}$ \\
\hline \multirow{2}{*}{$\begin{array}{l}\text { Lantelme (1994) } \\
\text { Toledo e Oprime } \\
(1996)\end{array}$} & Indicadores de qualidade & $\begin{array}{l}\text { Relacionados à medição da eficácia da empresa em atender } \\
\text { as necessidades e satisfação dos clientes. }\end{array}$ \\
\hline & $\begin{array}{l}\text { Indicadores de } \\
\text { produtividade }\end{array}$ & $\begin{array}{l}\text { Representam a eficiência do processo na obtenção dos } \\
\text { resultados esperados. Devem viabilizar coleta e análise de } \\
\text { dados de forma simples e direta, com medições ordenadas } \\
\text { e periódicas. }\end{array}$ \\
\hline \multirow{3}{*}{$\begin{array}{l}\text { Alárcon et al. (2001) } \\
\text { (indicadores } \\
\text { associados à } \\
\text { realização de } \\
\text { benchmarking) }\end{array}$} & Indicadores variáveis & $\begin{array}{l}\text { Indicam as decisões estratégicas e decisões que não são } \\
\text { referentes aos processos, mas que afetam o desempenho } \\
\text { da empresa ou do empreendimento. }\end{array}$ \\
\hline & Indicadores de processo & $\begin{array}{l}\text { Possuem o objetivo de medir o desempenho dos processos } \\
\text { mais importantes da empresa e do empreendimento, como } \\
\text { projetos, execução, planejamento e compras. }\end{array}$ \\
\hline & $\begin{array}{l}\text { Indicadores de } \\
\text { resultados }\end{array}$ & $\begin{array}{l}\text { Tentam medir o nível de sucesso atingido pelo } \\
\text { empreendimento. }\end{array}$ \\
\hline \multirow[t]{2}{*}{ Oliveira et al. (1995) } & $\begin{array}{l}\text { Indicadores estratégicos } \\
\text { ou gerenciais }\end{array}$ & $\begin{array}{l}\text { São estabelecidos com o objetivo de acompanhar e } \\
\text { impulsionar a implantação de estratégias. }\end{array}$ \\
\hline & Indicadores operacionais & $\begin{array}{l}\text { São estabelecidos em função dos objetivos e tarefas } \\
\text { desenvolvidos dentro de cada processo, devendo ser } \\
\text { coerentes com objetivos e estratégias adotadas pela } \\
\text { empresa. }\end{array}$ \\
\hline \multirow{2}{*}{$\begin{array}{l}\text { Hronec (1994) e } \\
\text { Lantelme et al. } \\
(2001)\end{array}$} & Indicador de produto & $\begin{array}{l}\text { Avalia se as características do produto atendem aos } \\
\text { requisitos do cliente previamente estabelecidos. }\end{array}$ \\
\hline & Indicador de processo & $\begin{array}{l}\text { Avalia se as características do processo atendem as } \\
\text { necessidades do cliente. Refere-se aos indicadores para } \\
\text { monitoramento de processos críticos da empresa e do } \\
\text { empreendimento, seja avaliando atendimento aos requisitos } \\
\text { do processo em si, ou do produto gerado. }\end{array}$ \\
\hline
\end{tabular}


Sutter (2002) $)^{2}$ apud Rozados (2005) propõe quatro critérios para seleção de indicadores de desempenho:

- Pertinência: um indicador deve ser fiel com o mínimo de distorção, deve ser justo ou estável, dar uma informação exata e renovável, e ser preciso;

- Caráter operacional: um indicador deve ser fácil de estabelecer, de utilizar, e aceitável, ou seja, não deve ser contrário à cultura da organização e deve permitir comunicação de diferentes partes envolvidas;

- Caráter consolidável (agregável): um indicador deve ser confiável, consolidável e agregável, ou seja, sua consolidação facilita as análises e as sínteses passadas, e permite deixar em evidência as tendências;

- Caráter econômico: um indicador deve ser calculável monetariamente ou ser útil à prevenção.

Em relação à definição de indicadores, Cândido et al. (2016), afirmam que o modelo apresentado por Neely et al. (1997) ${ }^{3}$ é o mais bem estruturado e pode ser utilizado como referência pela indústria da construção, no qual são apresentadas informações necessárias para a operacionalização dos indicadores: Título; Objetivo da medida; Relação com os objetivos; Meta; Fórmula; Frequência de medição; Frequência de revisão; Responsável pela coleta; Fonte dos dados; Encarregado, e o que ele deve fazer; Quem atua sobre os dados, e o que ele deve fazer; e Observações e comentários.

Outra questão relevante a ser considerada é quantidade de indicadores a ser adotada. Segundo Plentz (2014), 20 indicadores é o número recomendado pela literatura para um sistema equilibrado, pois, um número muito pequeno não daria visibilidade aos indicadores, e por outro lado, é necessária uma quantidade que não dificulte a implementação, bem como a coleta e análise dos dados, que demandam tempo e recursos.

\subsection{Desempenho na construção civil e no processo de projetos}

$\mathrm{Na}$ construção civil, a medição de desempenho se insere num campo de pesquisa diversificado. De acordo com Deng et al. (2012), por meio de estudos de outros autores na literatura, três objetivos podem ser identificados em nível geral:

- Propósito da Indústria: que avalia o desempenho do setor da construção a nível nacional e internacional;

- Finalidade de negócio: que mede o desempenho da organização, incluindo uma única avaliação e a medição contínua;

- Objetivo do projeto: que permite avaliar o desempenho e sucesso de projetos de construção.

A partir dessa análise, observa-se que a medição de desempenho no nível do negócio tem sido falha por falta de feedback adequado, disseminação e incorporação das lições aprendidas para gerar melhorias no âmbito do projeto e da construção. Já no nível do projeto pode funcionar ao longo do ciclo de vida de cada um, mas na maioria das empresas é insuficiente para induzir oportunidades de melhoria em longo prazo (DENG et al., 2012).

De acordo com Ali et al. (2012), a pressão externa sobre as empresas da construção para melhorar continuamente a sua produção e desempenho, tem resultado em diversos estudos no âmbito do projeto, no entanto, a demanda por avaliação de desempenho ao nível da empresa aumentou. Os autores apontam que indicadores financeiros tradicionais não podem ser o único determinante de

\footnotetext{
${ }^{2}$ SUTTER, É. Documentation, Information, Connaissances: la gestion de la qualité. Paris: ABFF 2002. (Collection Sciences de l'Information. Série Ètudes et Techiniques).

${ }^{3}$ NEELY, A.; RICHARDS, H.; MILLS, J.; PLATTS, K.; BOURNE, M. Designing Performance Measures: a structured approach. International Journal of Operations \& Production Management, v. 17, n. 11, p. 1131-1152, 1997.
} 
sucesso, ao passo que indicadores relacionados à satisfação do cliente externo, eficiência e eficácia do planejamento são cada vez mais importantes.

Quando se trata de sistemas de medição de desempenho, poucos estudos são encontrados com foco em empresas de projeto, como pouco se investiga o impacto dos projetos no desempenho da construção em geral, ainda que haja conhecimento da vinculação do projeto ao custo da construção desde o primeiro estudo de viabilidade econômica do empreendimento (MORAES et al., 2016).

Desse modo, a evidente carência de pesquisas voltadas para empresas de projeto de arquitetura conduziu a uma busca por trabalhos que abordam o SMD sob diferentes ópticas para a estruturação da proposta de SMD deste artigo. Da bibliografia estudada, quatro trabalhos foram selecionados, de forma que reúnem indicadores diversificados e abrangem os três objetivos gerais apresentados, cujos autores são: Jin et al. (2013), Bingol e Polat (2017), Orihuela et. al (2016) e Plentz (2014).

Jin et al. (2013) propõem um quadro prático para medir o desempenho de empresas de construção internacionais, destacando que o desempenho dessas empresas não é definido pelo sucesso do processo de internacionalização, nem pelo sucesso em contemplar projetos de construção internacionais, mas pelo apoio interno em conjunto com negócios internacionais que contribuem para o desempenho sustentado de toda empresa.

Assim, há uma contribuição não só para a internacionalização quanto para a medição de desempenho na indústria da construção como um todo, com a proposta de 27 indicadores organizados em seis dimensões: financeiro, mercado, clientes, processos internos do negócio, stakeholders, e por fim aprendizagem e crescimento.

Enquanto Jin et al. (2013) se concentram no objetivo mais geral, Bingol e Polat (2017) direcionam os esforços para a finalidade do negócio a partir da avaliação de pessoas envolvidas, com o desenvolvimento de uma estrutura de medição de desempenho gerencial baseada no modelo dos principais indicadores de desempenho (Key Performance Indicators - KPIs). Para os autores, o sucesso de um projeto depende diretamente do desempenho dos subcontratados, uma vez que são os responsáveis pela execução de grande parcela das atividades da construção, e que o desempenho dos candidatos a subcontratados deve ser medido e avaliado durante o processo de seleção pelos empreiteiros.

No âmbito do objetivo do projeto, Orihuela et al. (2016) apresentam 24 indicadores de resultado num quadro biaxial, estabelecidos pelo ciclo de vida do projeto que vai até a construção (estrutura vertical), e por cinco perspectivas (estrutura horizontal), das quais três são baseadas em critérios de sucesso de um projeto: qualidade, prazo e custo, e dois critérios de sustentabilidade adicionados: meio ambiente e sustentabilidade. Vale ressaltar que apesar da contribuição no estudo do desempenho de projetos, tal proposta pode atingir um nível de complexidade que poderia dificultar ou inviabilizar o sistema, pelo fato de depender diretamente do feedback de outros setores externos à empresa.

Há ainda trabalhos focados em avaliar a competitividade da empresa, como o de Plentz (2014), que apresenta um sistema de indicadores de gestão do design voltado para empresas desenvolvedoras de produtos, totalizando 20 indicadores, organizados em cinco categorias: qualidade, eficiência, inovação, resposta ao consumidor e resultado.

Apesar das empresas de arquitetura se diferirem por serem prestadoras de serviços, como projetos, há uma correlação com o modelo apresentado por Plentz (2014) pelas categorias e pela gestão do design, que não é incorporada na constituição dos SMDs adotados pelos demais setores da construção civil. 
As perspectivas qualidade e eficiência se destacam das demais, por serem aplicáveis tanto em nível do processo quanto do resultado final. No caso de projetos, para a escolha dos indicadores relacionados a essas duas perspectivas, é pertinente a consideração de aspectos presentes na prática das organizações, como a existência de atrasos de projeto, que ocorrem por diversos motivos, inclusive pela revisão frequente em decorrência de não conformidades ou mudança de escopo por parte do contratante, e também as dificuldades na interface entre disciplinas e agentes envolvidos.

Segundo Nóbrega Júnior e Melhado (2013), a qualidade do projeto é fundamental por determinar quase toda a competitividade e os custos do empreendimento, considerando que a partir de sua conclusão, há uma significativa redução na possibilidade de intervenção na obra e na lucratividade do negócio. Nesse contexto, o projeto assume um papel importante não só na empresa desenvolvedora, mas em todo o setor da construção civil.

Em relação ao gerenciamento de projetos, as empresas têm consciência dos fatores críticos de sucesso dos projetos, da importância do uso das metodologias de gerenciamento e da eficiência do uso dos indicadores de desempenho, mas ainda assim poucas utilizam destas técnicas e ferramentas no cotidiano de forma eficiente. Diante dessa realidade, os índices de insucesso nesse contexto são altos e os métodos de medição de desempenho ainda são pouco utilizados (BERNARDI, 2013).

Por outro lado, é demonstrado por meio de estudos de caso que projetos podem ser mais bem gerenciados com a utilização de indicadores, que consequentemente contribuem para a obtenção de sucesso do projeto e monitoramento das áreas consideradas como fatores críticos de sucesso, dos quais é do escopo que decorrem grande parte dos problemas em projetos pela falta de planejamento e controle. Além de possibilitar que os gerentes ajam de forma rápida e precisa ao tomar ações preventivas ou corretivas (BERNARDI, 2013).

Perante o exposto, convém enfatizar a relevância do papel dos stakeholders para o bom funcionamento de um SMD. O termo stakeholders é simplificadamente conhecido como partes interessadas e, de acordo com Mitchell et al. (1997), os tipos de entidades que podem ser consideradas stakeholders são pessoas, grupos, organizações, instituições, sociedades e o ambiente natural. Na esfera do projeto, Bernardi (2013) afirma que para um projeto ser considerado com sucesso não basta que o mesmo seja concluído dentro do prazo e dos custos planejados, a visão dos patrocinadores é muito importante para conceituá-lo como tal.

Por fim, a multiplicidade de pontos a ser levantada e analisada para a proposição de um SMD tanto para uma empresa de arquitetura quanto para outras empresas no setor da construção, torna clara a necessidade de compreender a importância e o porquê de cada indicador individualmente, bem como avaliar a pertinência da sistematização e criação de categorias de acordo com os objetivos estratégicos de cada empresa, numa estrutura que seja viável e útil.

\section{Método}

Para coleta dos dados, optou-se por entrevista semiestruturada e questionário com o objetivo de analisar a visão da gerência da empresa sobre o sistema de desempenho atual e suas necessidades.

\subsection{Classificação da pesquisa}

A presente pesquisa possui abordagem qualitativa, uma vez que busca aprofundar-se na compreensão do tema, assim como da organização escolhida para estudo de caso. Sob o ponto de vista de sua natureza, é aplicada, pois objetiva gerar conhecimentos voltados à solução de problemas específicos para futura aplicação na prática. 
Quanto aos objetivos é classificada como exploratória, utilizando-se da interação teórico-prática, a partir do levantamento bibliográfico e coleta de dados, com a finalidade de desenvolver uma estrutura de análise que facilite a identificação dos indicadores relevantes para a proposição do sistema.

\subsection{Delineamento da pesquisa}

A pesquisa divide-se em três grandes fases, subdivididas em oito etapas, sendo que a revisão bibliográfica permeia todo o trabalho, com ênfase maior na primeira fase (Figura 1).

Figura 1: Delineamento da pesquisa.

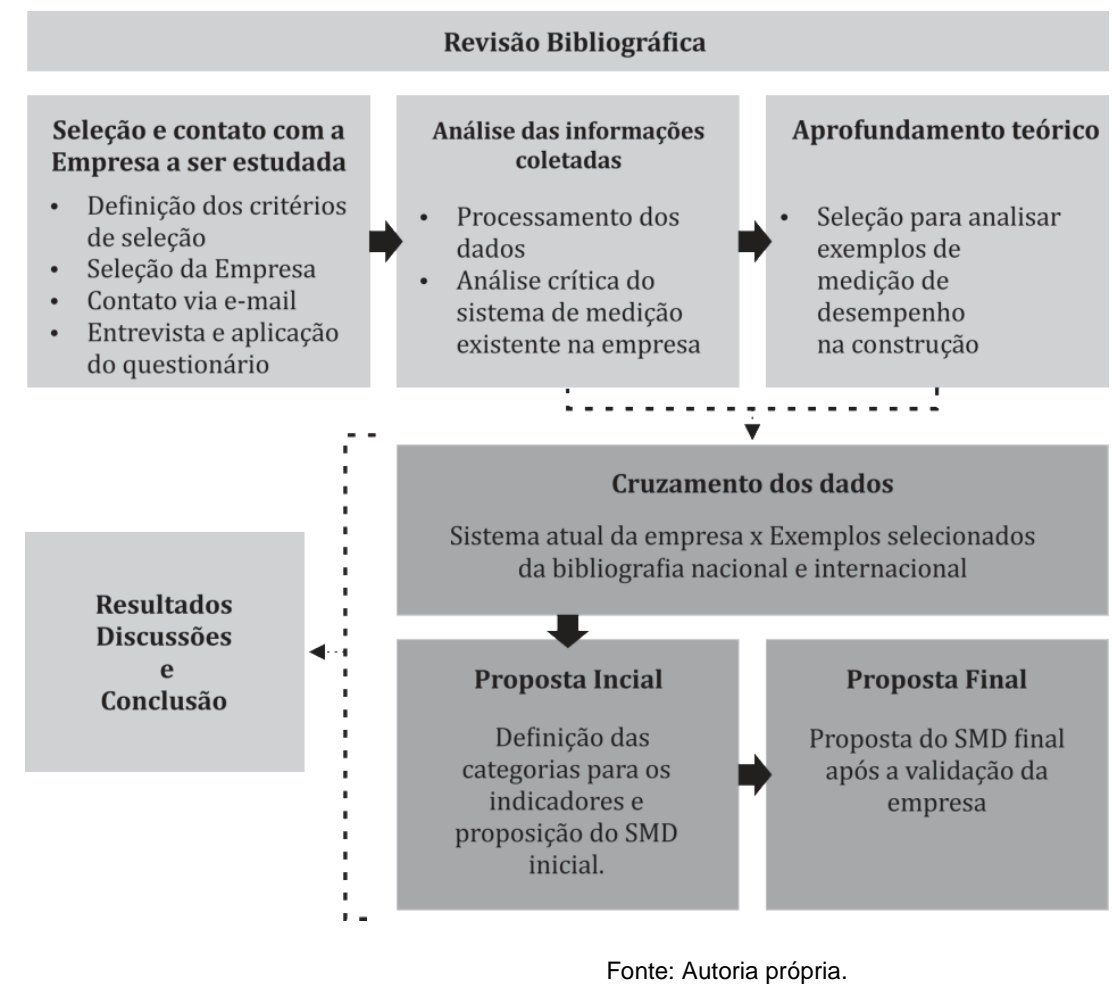

\subsubsection{Critérios de seleção da empresa e contato}

Os critérios de seleção da empresa para estudo de caso na pesquisa foram: possuir um Sistema de Gestão da Qualidade consolidado; possuir relevância no mercado; ter como atividade principal o desenvolvimento de projetos; e interesse por parte da empresa em colaborar com a pesquisa.

Após a definição dos critérios, a forma de contato ocorreu via e-mail, com o envio da carta convite para parceria de pesquisa com a Universidade Federal de Goiás (UFG) e também via contato telefônico.

\subsubsection{Levantamento de dados}

Para o levantamento de dados aplicou-se um questionário e uma entrevista semiestruturada a um dos membros da diretoria, a fim de conhecer o perfil e características da empresa, bem como ter acesso a documentos referentes ao sistema de medição de desempenho e ao gerenciamento de processos e projetos.

Após o levantamento, definiu-se a forma de apresentação dos dados em cada etapa. Primeiramente, foi estruturado o SMD atual da empresa (Tabela 2), para entendimento de como se dá o funcionamento e organização dos indicadores, os quais estão dispostos em quatro categorias intituladas metas, sendo estas as metas globais da empresa. O SMD da empresa está associado ao Sistema de Gestão da Qualidade e relacionado com a Política da Qualidade da empresa. 
Tabela 2: Modelo da estrutura do SMD atual da Empresa.

\begin{tabular}{l|l|l|l|l|l|l}
\hline Meta & Objetivos & Indicador & $\begin{array}{l}\text { Unid. } \\
\text { Medida }\end{array}$ & Como medir & $\begin{array}{l}\text { Freq. da } \\
\text { medição }\end{array}$ & Responsabilidade \\
\hline & \multicolumn{7}{|c}{ Fonte: Adaptado da Empresa. } & & \\
\hline
\end{tabular}

Fonte: Adaptado da Empresa.

O campo "Objetivos" se destina aos itens que devem ser medidos, desempenhando o papel dos próprios indicadores. Já o título "Indicador" se refere à meta a ser alcançada com cada item, dada em percentual ou número. Assim, esta organização apresenta algumas divergências em relação a exemplos encontrados na bibliografia, e por esse motivo, optou-se por simplificar a apresentação dos dados, objetivando maior clareza.

Tal simplificação resultou na criação de um quadro com a categorização dos indicadores, suas respectivas siglas que permitem a identificação dos indicadores a serem mantidos na proposta desta pesquisa, uma vez que podem mudar de categoria ou nomenclatura, e por último a quantidade por categoria. Para os doze indicadores foram atribuídas sete categorias: satisfação dos clientes, capacitação das equipes, qualidade, eficiência, finanças, sustentabilidade e inovação (Tabela 3).

Tabela 3: Modelo da categorização dos indicadores da Empresa.

\begin{tabular}{|c|c|c|c|}
\hline Categorias & Sigla & Indicadores & Qtd. \\
\hline \multirow{3}{*}{$\begin{array}{l}\text { Satisfação dos } \\
\text { clientes }\end{array}$} & Id1 & & \\
\hline & Id2 & & \\
\hline & Id3 & & \\
\hline \multirow{3}{*}{$\begin{array}{l}\text { Capacitação das } \\
\text { equipes }\end{array}$} & Id4 & & \\
\hline & Id5 & & \\
\hline & Id6 & & \\
\hline \multirow{2}{*}{ Qualidade } & Id7 & & \\
\hline & Id8 & & \\
\hline Eficiência & Id9 & & \\
\hline Finanças & Id10 & & \\
\hline Sustentabilidade & Id11 & & \\
\hline Inovação & Id12 & & \\
\hline
\end{tabular}

Fonte: Autoria própria.

Para a proposta do SMD, optou-se por apresentar um quadro para cada categoria estabelecida, contendo os indicadores, suas siglas e a fonte de cada indicador, sendo da empresa ou da literatura, como exemplifica a Tabela 4. Dessa forma, cada quadro é apresentado juntamente com os textos explicativos, para tornar claro o processo de construção do sistema inicial, com o porquê de cada categoria criada.

Tabela 4: Modelo da categorização dos indicadores para a proposta.

\begin{tabular}{c|l|l}
\hline \multicolumn{2}{l}{ Categoria } & \\
\hline Sigla & Indicador & Fonte \\
\hline Id $\left(\mathrm{n}^{\circ}\right)$ & Indicador existente na empresa & Empresa \\
\hline- & Indicador novo proposto & Autor (ano) \\
\hline
\end{tabular}

Fonte: Autoria própria.

Posteriormente, criou-se um novo quadro para a proposta completa do SMD, com a finalidade de fornecer uma visão geral mais detalhada, contendo os seguintes itens: Categoria; Indicador; Fórmula; Unidade de medida; e Frequência da medição (Tabela 5).

Tabela 5: Modelo do Sistema de Medição de Desempenho completo.

\begin{tabular}{l|l|l|l|l}
\hline Categoria & Indicador & Fórmula & Un. & Freq. \\
\hline & & & & \\
\hline \multicolumn{2}{c|}{ Fonte: Autoria própria. }
\end{tabular}




\subsubsection{Caracterização da empresa}

A empresa selecionada para o estudo de caso localiza-se em Goiânia, atua no mercado há quinze anos, destacando-se pela experiência em projetos arquitetônicos de edifícios de múltiplos pavimentos, cujo portfólio engloba projetos residenciais, comerciais, uso misto, urbanismo, dentre outros.

As incorporadoras e construtoras são os principais clientes da empresa. Os serviços ofertados pela empresa vão do Briefing à entrega do Projeto Executivo e Detalhamento, e ainda quando contratado, o acompanhamento da execução do projeto. Após a definição do produto, a análise da Viabilidade Técnica é ofertada sem custos, como parte da estratégia para captação de clientes. Os demais serviços desenvolvidos a partir da contração são: Anteprojeto e Projeto Legal de Arquitetura, Projeto Executivo de Arquitetura, Projeto de Especificações e Detalhamentos, e Coordenação de Projetos Complementares.

Desde 2011, a empresa possui Certificação ISO 9001:2008 pelo Instituto de Certificação Qualidade Brasil (ICQ Brasil), o que conduziu à criação da Política de Qualidade, com a definição de algumas premissas, como manter a qualidade do serviço por meio da melhoria contínua de modo a proporcionar a satisfação dos clientes, juntamente com o desenvolvimento sustentável e projetos diferenciados. Consequentemente, ferramentas de medições e monitoramentos para os processos foram adotadas, dentre elas, documentos de medições, checklists, indicadores de desempenho, e ainda o software Wrike ${ }^{4}$ para apoio na coordenação de projetos.

Classificada como pequeno porte, atualmente a empresa conta com uma equipe de doze funcionários contratados e um terceirizado. A estrutura organizacional é tida como matricial, na qual todos os processos são organizados de acordo com os departamentos existentes. A gestão de projetos pode ser considerada como o cerne da empresa, visto que dez funcionários são designados a essa parte, sendo eles arquitetos e um estagiário, e para os demais departamentos ligados ao setor administrativo tem-se dois funcionários; todos com supervisão e coordenação da diretoria. A estrutura da empresa é apresentada no organograma a seguir (Figura 2).

Figura 2: Organograma da Empresa.

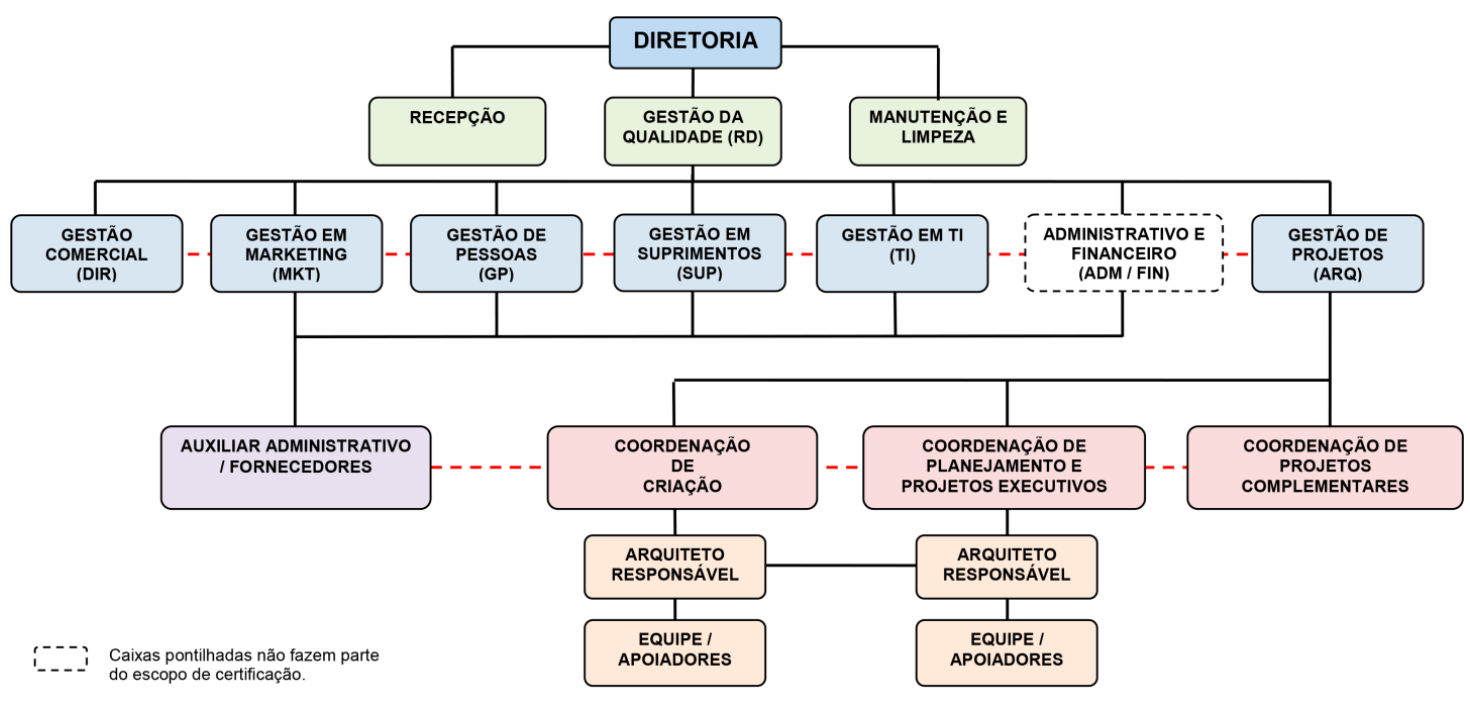

Fonte: Empresa.

\footnotetext{
4 Wrike é um software de gestão de projetos em tempo real, que oferece a possibilidade da atuação de diferentes equipes a partir de um único sistema, e assim monitorar processos e resultados.

Fonte: Wrike, 2018. Disponível em: https://www.wrike.com/pt-br//. Acessado em: 05 de setembro de 2018.
} 


\section{Análise dos dados e discussões}

Após a coleta e processamento dos dados, são apresentados os resultados obtidos para discussão acerca da realidade da empresa, subsidiando a próxima fase da proposição do sistema de medição de desempenho (SMD). Desse modo, partiu-se da ideia de entender a dinâmica da organização e seus controles existentes.

O SMD da empresa teve seu início em 2012 após a implementação do Sistema de Gestão da Qualidade (SGQ). A primeira certificação ocorreu em 2011 e foi renovada em 2014, o que decorreu na atualização e correção nos métodos de controles abrangidos pela gestão da empresa. Até o período da coleta dos dados, a certificação não havia sido renovada, destacando-se a necessidade de revisão dos documentos relacionados ao SGQ, uma vez que todo sistema atende à norma NBR ISO 9001:2008.

A Política de Qualidade da empresa pode ser vista como a estrutura norteadora de todo o processo de medição de desempenho, uma vez que os indicadores são categorizados de acordo com as quatro metas globais da empresa, com a finalidade de monitorar se as mesmas estão sendo atingidas.

Nota-se que há ausência de indicadores com foco em atividades e processos específicos que são críticos para o desempenho da empresa. Ainda que a maioria dos processos possuam medição e monitoramento, muitos não são tabulados e convertidos em indicadores dentro do SMD. Ou seja, há uma descentralização de informações, dificultando análises mais críticas e comparação dos resultados com períodos anteriores.

Primeiramente, os indicadores foram extraídos do documento original e agrupados de acordo com as categorias identificadas, que foram sete no total: satisfação dos clientes, capacitação das equipes, qualidade, eficiência, finanças, sustentabilidade e inovação, juntamente com as siglas de cada indicador e a quantidade por categoria (Tabela 6).

Tabela 6: Indicadores atuais da Empresa.

\begin{tabular}{l|c|l|c}
\hline Categorias & Sigla & Indicadores & Qtd. \\
\hline \multirow{2}{*}{$\begin{array}{l}\text { Satisfação } \\
\text { clientes }\end{array}$} & Id1 & Satisfação do cliente contratante & \multirow{2}{*}{3} \\
\cline { 2 - 4 } & Id2 & Satisfação do cliente contratante (obra) & \\
\cline { 2 - 4 } & Id3 & Satisfação do cliente usuário & \multirow{2}{*}{3} \\
\hline \multirow{2}{*}{$\begin{array}{l}\text { Capacitação das } \\
\text { equipes }\end{array}$} & Id4 & Atingir eficácia com os treinamentos internos e externos oferecidos à equipe & \multirow{2}{*}{2} \\
\cline { 2 - 5 } & Id5 & Fornecer curso de gestão para os diretores & 1 \\
\cline { 2 - 5 } & Id6 & Promover a participação dos diretores e coordenadores em eventos & 1 \\
\hline \multirow{2}{*}{ Qualidade } & Id7 & Melhorias nos processos produtivos & 1 \\
\cline { 2 - 5 } & Id8 & Projetos com qualidade técnica, funcional, estética, gráfica e executiva. & 1 \\
\hline Eficiência & Id9 & Entregar Projetos no prazo planejado, por fases de projeto. & \\
\hline Finanças & Id10 & Lucratividade anual & \\
\hline Sustentabilidade & Id11 & Projetos com soluções voltadas para sustentabilidade & \\
\hline Inovação & Id12 & Projetos com itens de inovação & \\
\hline
\end{tabular}

Fonte: Autoria própria.

Das sete categorias, nota-se que "satisfação dos clientes" e "capacitação das equipes" são as mais representativas, contando com três indicadores cada uma. Em seguida, a qualidade, com dois indicadores, e as demais somente com um. Desse modo, identifica-se como dado crucial nessa pesquisa, a pequena quantidade de indicadores voltados para monitoramento e controle da atividade principal da empresa, o desenvolvimento de projetos, assim como para seus processos e a eficiência da equipe. $O$ que sugere a necessidade de mudanças de forma a incluir o processo de projeto como parte fundamental do sistema de medição.

Dos controles existentes e não incluídos no documento de Política da Qualidade, e que também não são tabulados, estão o controle de riscos, por meio dos estudos de viabilidade técnica realizados; o 
atendimento à norma NBR 15575: 2013, cujo documento não foi atualizado após o amadurecimento das necessidades da empresa; e o controle de horas trabalhadas por projeto, que não possui relação direta com o cumprimento dos prazos de projeto, inviabilizando a mensuração dos prejuízos decorrentes das mudanças de escopo.

Compreende-se que o sistema de indicadores atual da empresa é estruturado e revela um grande potencial para melhorias, considerando que diversas ferramentas de acompanhamento de projetos e controle de processos já são adotadas, porém nem todos são trabalhados em conjunto no SMD. Consequentemente, o mesmo poderia ser reformulado e aperfeiçoado, de modo a ser entendido por todos os usuários das informações, inclusive pela equipe de apoiadores, que atualmente tem acesso aos dados em reuniões anuais.

Contudo, a proposição de um novo SMD além de possibilitar um avanço para a empresa em questão, poderá contemplar as principais esferas necessárias para medição de desempenho em outras empresas de projetos, principalmente de arquitetura, e consequentemente trazer contribuições para o setor da construção em geral.

\section{Proposição do sistema de medição de desempenho}

Após as análises sobre o SMD da empresa, foi realizado o cruzamento desses dados com a bibliografia estudada acerca das iniciativas nacionais e internacionais, para ponderação de quais indicadores permaneceriam na proposição e quais seriam acrescentados.

Uma primeira avaliação levou em consideração o alinhamento dos indicadores com os objetivos estratégicos da empresa, a pertinência e demais critérios de seleção de indicadores apresentados anteriormente, sugeridos por Sutter (2002) apud Rozados (2005): pertinência, caráter operacional, consolidável e econômico.

Posteriormente, foi realizada uma avaliação sobre as categorias estabelecidas na etapa de análise e discussão dos dados, com o objetivo de reduzir as sete para um número capaz de proporcionar uma hierarquia e melhor organização, sem desprezar o foco existente de cada uma.

Para a proposição do SMD, buscou-se uma composição de indicadores de diferentes classificações: específicos e globais, de produtividade ou eficiência, de processo e de resultados. De modo a abarcar as diversas esferas que possuem relevância para o desempenho da organização como um todo.

A proposta final resultou em cinco categorias: stakeholders, eficiência, qualidade, inovação e sustentabilidade, e resultado. Cada uma com quatro indicadores, contando com os novos que foram escolhidos por meio de exemplos na literatura e os já existentes que foram mantidos ou adaptados. Nenhum dos indicadores existentes foi desconsiderado.

Apesar de haver diferenciação na abordagem sobre sistemas de medição e indicadores de desempenho entre as referências utilizadas, todas se relacionam com o contexto da empresa estudada por possuírem indicadores no âmbito da construção civil, do projeto e do design. As quais foram: Bingol e Polat (2017), Orihuela et al. (2016), Plentz (2014) e Jin et al. (2013).

\subsection{Categoria Stakeholders}

A categoria stakeholders (Tabela 7) compreende indicadores relacionados às partes interessadas, internas e externas à empresa, que possibilitam influenciar o comportamento. 
Tabela 7: Categoria Stakeholders.

\begin{tabular}{c|c|l|l}
\hline & Sigla & Indicador & Fonte \\
\hline \multirow{3}{*}{ Externos } & Id1 & Satisfação do cliente contratante & Empresa \\
\cline { 2 - 4 } & Id3 & Satisfação do cliente final (usuário) & Empresa \\
\hline \multirow{3}{*}{ Internos } & - & Satisfação da equipe & Jin et al., 2013 \\
\cline { 2 - 4 } & Id4 e 5 & Treinamentos internos e externos & Empresa \\
\hline
\end{tabular}

Fonte: Autoria própria.

Quanto aos stakeholders externos, foram mantidos dois indicadores existentes (Id1 e Id3) que avaliam o grau de satisfação dos clientes quanto à empresa, qualidade do serviço prestado e aos projetos.

Para os internos, foi proposto um indicador a partir da fusão de dois já praticados pela empresa: "Atingir eficácia com os treinamentos internos e externos oferecidos à equipe" (Id4) e "Curso de gestão para os diretores" (Id5). Essa integração resultou no indicador "Treinamentos internos e externos", com o intuito de ser uma medida mais objetiva que abrange toda equipe. Pois, o controle da eficácia dos treinamentos medido pela produtividade da equipe pode resultar em dados subjetivos, uma vez que a produtividade de cada funcionário pode ser influenciada por outros fatores.

A satisfação da equipe foi contemplada utilizando-se como referência o indicador "satisfação do funcionário" proposto por Jin et al. (2013), que inclui responsabilidades, oportunidades justas, treinamento, planejamento de carreira e remuneração. Além dessas características, sugere-se que outras questões destacadas por Roncon et al. (2015) sejam incluídas na avaliação de satisfação, como as relativas ao clima organizacional adequado, uma vez que a valorização das pessoas proporciona meios para realização pessoal e valor para a organização.

\subsection{Categoria Eficiência}

A categoria eficiência tem por objetivo medir a eficiência dos processos na obtenção dos resultados esperados. Como pode ser observado na Tabela 8, apenas um indicador da empresa foi identificado para essa categoria, o de entrega dos projetos no prazo planejado (Id9). Para os demais, a escolha se deu a partir das necessidades da empresa analisadas anteriormente.

Tabela 8: Categoria Eficiência.

\begin{tabular}{l|l|l}
\hline Sigla & Indicador & Fonte \\
\hline Id9 & \% De projetos entregues no prazo planejado & Empresa \\
\hline- & \% De projetos que cumpriram o orçamento & Plentz, 2014 \\
\hline- & \% De horas de retrabalho & Plentz, 2014 \\
\hline- & Taxa de propostas bem-sucedidas & Bingol e Polat, 2017 \\
\hline
\end{tabular}

O indicador relacionado ao cumprimento do orçamento por projetos foi extraído e adaptado de Plentz (2014), cujo objetivo é avaliar o quão eficiente o setor de desenvolvimento de projetos está sendo no uso dos recursos destinados ao mesmo.

O indicador horas de retrabalho foi extraído da mesma pesquisa, porém retirado da categoria qualidade e incluído na categoria eficiência. Ainda que numa empresa de projetos o retrabalho esteja associado a revisões, não conformidades e, consequentemente a qualidade do projeto, optou-se por analisar este indicador quanto à produtividade da equipe, através da associação das horas de retrabalho com as horas totais trabalhadas nos projetos.

Por último, o indicador taxa de propostas bem-sucedidas, utilizado por Bingol e Polat (2017). No contexto da empresa estudada, sugere-se que esse indicador seja destinado às propostas de viabilidade técnica, que são apresentadas aos clientes sem custos, pois, além de possibilitar o 
gerenciamento de um risco, facilitará a análise do impacto dessa escolha sobre a produtividade da equipe.

\subsection{Categoria Qualidade}

A categoria qualidade objetiva medir a eficácia da empresa em atender suas necessidades e as dos clientes, podendo influenciar a satisfação dos mesmos por meio do alcance das metas estabelecidas, bem como avaliar sua competitividade através da qualidade dos serviços e projetos.

Como se observa na Tabela 9, os indicadores Id8 e Id2 foram mantidos da empresa, porém modificados. $O$ indicador "Porcentagem de cumprimento de Checklist" (Id8*) foi adotado utilizando o exemplo de Plentz (2014), visando atender às metas do antigo indicador Id8 da empresa: desenvolver projetos com qualidade técnica, funcional, estética, gráfica e executiva, cuja forma de medição atual se dá pelo percentual medido da qualidade técnica dos projetos. Ou seja, buscou-se tornar mais clara a demonstração dos resultados de processos que já são medidos pela empresa.

Tabela 9: Categoria Qualidade.

\begin{tabular}{l|l|l}
\hline Sigla & Indicador & Fonte \\
\hline Id8* & \% De cumprimento de Checklist & Empresa; Plentz, 2014 \\
\hline- & Índice de não conformidade em projeto & Orihuela et al., 2016* \\
\hline- & Índice de cumprimento da NBR 15575: 2013 & Orihuela et al., 2016* \\
\hline Id2* & Índice de reclamação do cliente contratante (obra) & Empresa \\
\hline
\end{tabular}

Fonte: Autoria própria.

Nota: * Indicadores adaptados

Já o indicador "Índice de reclamação do cliente contratante (obra)" foi construído a partir do indicador existente relacionado à satisfação desse cliente (Id2). Assim, deixa o foco da satisfação do cliente para focar na avaliação da qualidade do projeto. Pois os itens analisados por esse cliente específico, de acordo com especificação no SMD atual da empresa, estão diretamente relacionados à qualidade do projeto, quanto à clareza, compatibilidade de projetos e presteza na solução de problemas. Dessa forma, o objetivo da modificação é facilitar a identificação de possíveis falhas e tomada de ações preventivas e corretivas.

Para os outros dois indicadores, utilizou-se como referência o proposto por Orihuela et al. (2016), originalmente um indicador de conformidade dos parâmetros arquitetônicos, que mede padrões ou parâmetros de projeto relacionados aos aspectos legais que defendem os interesses dos usuários dos edifícios e da sociedade em geral.

O "Índice de não conformidade em projeto" verifica o quanto os métodos e processos estipulados foram atendidos, por meio da aplicação de checklists voltados ao projeto. E com objetivo semelhante, o indicador de índice de cumprimento da norma NBR 15575:2013, verifica o atendimento específico desta norma, por sua relevância em projetos de arquitetura e da construção civil em geral, e pela existência de uma ferramenta de controle presente na empresa com esse foco, que servirá como base para coleta dos dados.

\subsection{Categoria Inovação e sustentabilidade}

Inovação e sustentabilidade foram unidas nessa categoria, considerando a possibilidade de que ambas possam contribuir para a evolução dos processos e projetos, prever tendências e contribuir para o desenvolvimento sustentável.

Dos quatro indicadores, três fazem parte do SMD atual da empresa e foram mantidos. Para os dois primeiros (Id 11 e Id12), como se observa na Tabela 10, não houve mudanças. Já o de participação de diretores e coordenadores em eventos (Id6), anteriormente com foco na capacitação da equipe, foi inserido nessa categoria com a perspectiva de direcionar essa participação para atualização da 
empresa sobre novas práticas no setor da construção, bem como se situar diante as inovações praticadas por outras empresas no mercado.

Tabela 10: Categoria Inovação e Sustentabilidade.

\begin{tabular}{l|l|l}
\hline Sigla & Indicador & Fonte \\
\hline Id12 & Número de inovações em projetos & Empresa \\
\hline Id11 & Número de soluções sustentáveis em projetos & Empresa \\
\hline Id6 & $\begin{array}{l}\text { Participação de diretores e coordenadores em eventos (de arquitetura, } \\
\text { engenharia e áreas afins) }\end{array}$ & Empresa \\
\hline- & Número de benchmarkings internos realizados & Jin et al., 2013 \\
\hline
\end{tabular}

Fonte: Autoria própria.

O quarto indicador foi elaborado a partir de Jin et al. (2013), e adaptado do original relacionado à partilha de informações e eficiência do conhecimento interno. Assim como o indicador anterior, este não se limita apenas ao âmbito da inovação e sustentabilidade, como por exemplo, também está relacionado à aprendizagem, porém, se encaixa adequadamente a esta categoria, visto que a transmissão de conhecimentos acerca de inovações e sustentabilidade entre coordenadores e diretores que participam de eventos e os demais membros da equipe é necessária, assim como é preciso uma conscientização de toda equipe quanto ao trabalho realizado e incentivo à participação de todos.

\subsection{Categoria Resultado}

Por fim, a categoria resultado avalia o nível de sucesso obtido nas finanças, bem como acompanha se as metas estratégicas estão sendo atingidas.

O indicador rentabilidade anual (Id 10) é o único financeiro utilizado pela empresa estudada (Tabela 11), e foi mantido por sua relevância. O segundo indicador, variação na receita, utilizado por Plentz (2014), também faz parte da perspectiva financeira e permite análises sobre a evolução da empresa em um período de tempo determinado.

Tabela 11: Categoria Resultado.

\begin{tabular}{l|l|l}
\hline Sigla & Indicador & Fonte \\
\hline Id10 & Rentabilidade anual & Empresa \\
\hline- & Variação na receita & Plentz, 2014 \\
\hline- & Participação de mercado & Plentz, 2014 \\
\hline Id7 & Melhoria nos processos produtivos & Empresa \\
\hline
\end{tabular}

Fonte: Autoria própria.

Como o benchmarking entre empresas de arquitetura é uma prática pouco relatada na literatura e pontuada pela empresa como ausente em seu contexto, uma das alternativas para avaliar o posicionamento da empresa se dá por meio da obtenção de dados do mercado imobiliário. Nesse sentido, utilizou-se o indicador "Participação de mercado", extraído de Plentz (2014).

Para finalizar a proposta dos indicadores, o Id7, que é "melhoria nos processos produtivos", foi mantido, por buscar a melhoria contínua dos processos e representar uma das principais estratégias da empresa. Ou seja, estando na categoria resultado se torna um indicador de maior peso ao invés de representar apenas a esfera operacional da empresa.

\subsection{Proposta do SMD}

O SMD foi sistematizado a partir da definição das categorias apresentadas e de seus respectivos indicadores. Após a conclusão da proposta do SMD, a mesma foi apresentada à direção da empresa participante do estudo de caso, na busca de uma validação e obtenção de feedback para 
aprimoramento dos indicadores. Dessa forma, todas as observações foram consideradas e a revisão resultou na proposta final com o SMD reformulado (Tabela 12).

Tabela 12: Sistema de Medição de Desempenho Final.

\begin{tabular}{|c|c|c|c|c|}
\hline Categoria & Indicador & Fórmula & Un. & Freq. \\
\hline \multirow{5}{*}{ 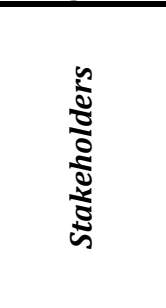 } & Satisfação do cliente contratante & $\begin{array}{l}\text { Avaliação de satisfação } \\
\text { contratante }\end{array}$ & $P$ & Semestral \\
\hline & $\begin{array}{l}\text { Satisfação do cliente contratante } \\
\text { (obra) }\end{array}$ & $\begin{array}{l}\text { Avaliação de satisfação do cliente } \\
\text { contratante da obra }\end{array}$ & $P$ & Semestral \\
\hline & Satisfação do cliente final (usuário) & Avaliação de satisfação do cliente final & $P$ & Semestral \\
\hline & Satisfação do engenheiro da obra & $\begin{array}{l}\text { Avaliação de satisfação quanto ao } \\
\text { serviço e projeto no contexto da obra }\end{array}$ & $\mathrm{P}$ & $\begin{array}{l}\text { Por } \\
\text { projeto }\end{array}$ \\
\hline & Satisfação da equipe & Avaliação de satisfação da equipe & $\mathrm{P}$ & Semestral \\
\hline \multirow{5}{*}{ : } & $\begin{array}{l}\text { Taxa de estudos de viabilidade } \\
\text { bem-sucedidos }\end{array}$ & $\begin{array}{l}\text { № de estudos de viabilidade bem- } \\
\text { sucedidos / № total de propostas } \\
\text { apresentadas * } 100\end{array}$ & № & Semestral \\
\hline & $\%$ De atendimento ao briefing & $\begin{array}{l}\text { Itens do briefing atendidos em projeto / } \\
\text { Total de itens do briefing * } 100\end{array}$ & № & $\begin{array}{l}\text { Por } \\
\text { projeto }\end{array}$ \\
\hline & $\begin{array}{l}\text { \% De horas de retrabalho (motivos } \\
\text { internos) }\end{array}$ & $\begin{array}{l}\mathrm{H} \text { de retrabalho por motivos internos / } \mathrm{h} \\
\text { trabalhadas } * 100\end{array}$ & $\mathrm{H}$ & $\begin{array}{l}\text { Por } \\
\text { projeto }\end{array}$ \\
\hline & $\begin{array}{l}\text { \% De horas de retrabalho (motivos } \\
\text { externos) }\end{array}$ & $\begin{array}{l}\text { H de retrabalho por motivos externos / } \mathrm{h} \\
\text { trabalhadas } * 100\end{array}$ & № & $\begin{array}{l}\text { Por } \\
\text { projeto }\end{array}$ \\
\hline & $\begin{array}{l}\% \text { De projetos entregues no prazo } \\
\text { planejado }\end{array}$ & $\begin{array}{l}\text { Número de projetos entregues no prazo } \\
\text { / total de projetos entregues } * 100\end{array}$ & & Semestral \\
\hline \multirow{4}{*}{$\begin{array}{l}\frac{0}{\pi} \\
\frac{\pi}{\pi} \\
\frac{\pi}{\tilde{z}} \\
\frac{\pi}{2}\end{array}$} & Treinamentos internos e externos & № de treinamentos realizados & № & Anual \\
\hline & $\begin{array}{l}\text { Índice de não conformidade em } \\
\text { projeto (de acordo com itens dos } \\
\text { checklists) }\end{array}$ & $\begin{array}{l}\text { № de não conformidades / № de } \\
\text { parâmetros aplicáveis }\end{array}$ & № & $\begin{array}{l}\text { Por } \\
\text { projeto }\end{array}$ \\
\hline & $\begin{array}{l}\text { Índice de cumprimento da NBR } \\
\text { 15575: } 2013\end{array}$ & $\begin{array}{l}\text { № de itens atendidos / № parâmetros } \\
\text { aplicáveis }\end{array}$ & № & $\begin{array}{l}\text { Por } \\
\text { projeto }\end{array}$ \\
\hline & Melhoria nos processos produtivos & № de melhorias implantadas & № & Semestral \\
\hline \multirow{4}{*}{ 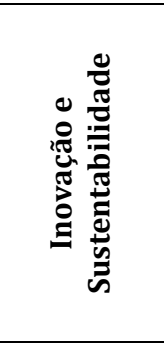 } & Investimento em P\&D sobre receita & Investimentos em P\&D / receita *100 & $\$$ & Anual \\
\hline & $\begin{array}{l}\text { Número de soluções sustentáveis } \\
\text { em projetos }\end{array}$ & $\begin{array}{l}\text { № de itens de sustentabilidade nos } \\
\text { projetos }\end{array}$ & № & Semestral \\
\hline & $\begin{array}{l}\text { Participação de diretores } \mathrm{e} \\
\text { coordenadores em eventos (de } \\
\text { arquitetura, engenharia e áreas } \\
\text { afins) }\end{array}$ & № de eventos participados & № & Anual \\
\hline & $\begin{array}{l}\text { Número de benchmarkings internos } \\
\text { realizados }\end{array}$ & № de benchmarkings internos realizados & № & Semestral \\
\hline \multirow{5}{*}{ Resultado } & $\begin{array}{l}\text { Taxa de propostas financeiras } \\
\text { fechadas }\end{array}$ & $\begin{array}{l}\text { № de propostas financeiras fechadas / } \\
\text { Total de propostas financeiras } \\
\text { apresentadas } * 100\end{array}$ & $\$$ & Anual \\
\hline & Participação de mercado & $\begin{array}{l}\mathrm{N}^{\circ} \text { de empreendimentos, cujo projeto } \\
\text { arquitetônico foi realizado pela empresa } \\
\text { / No total de empreendimentos lançados } \\
\text { (de acordo com as tipologias que a } \\
\text { empresa trabalha) }\end{array}$ & № & Anual \\
\hline & Rentabilidade anual & Despesa / Receita * 100 & № & Anual \\
\hline & Variação na receita & $\begin{array}{l}\text { Receita período atual / Receita período } \\
\text { anterior* } 100\end{array}$ & $\$$ & Anual \\
\hline & Expansão do número de clientes & $\begin{array}{l}\text { № de clientes período anterior }-\mathrm{n}^{\circ} \text { de } \\
\text { clientes período atual }\end{array}$ & $\$$ & Anual \\
\hline
\end{tabular}

Fonte: Autoria própria.

Segundo a empresa, a proposição das categorias foi considerada adequada ao atendimento de suas necessidades, de modo que as cinco permaneceram com o mesmo foco anterior, apesar das modificações realizadas. Assim, o SMD resultou em 23 indicadores, sendo que os acrescentados podem ser opcionais de acordo com a necessidade de outras empresas. 
$\mathrm{Na}$ categoria stakeholders, acrescentou-se o indicador "Satisfação do cliente contratante (obra)", anteriormente intitulado de "índice de reclamação do cliente contratante (obra)" na categoria qualidade, uma vez que em determinados períodos pode não haver reclamações e o indicador teria mais utilidade com a avaliação da satisfação. Outra modificação foi a transferência de "treinamentos internos e externos" para a categoria qualidade.

Ainda na categoria stakeholders, foi acrescentado o indicador de satisfação do engenheiro de obra, com objetivo de estabelecer a interface projeto-obra de forma mais objetiva. Outros aspectos não incorporados no SMD final podem ser apontados como importantes nessa categoria, como a realização da avaliação de equipes externas à empresa, que são responsáveis pelos projetos complementares, bem como ter avaliação realizada pelas mesmas sobre a empresa para obtenção de feedback.

$\mathrm{Na}$ categoria eficiência, o indicador referente ao cumprimento do orçamento por projeto foi retirado, pelo fato do orçamento estar diretamente relacionado ao prazo na situação em que se cobra por hora trabalhada em cada projeto, como é o caso da empresa estudada. E acrescentou-se o atendimento ao briefing, para que todas as fases do projeto sejam monitoradas. Quanto à porcentagem de horas de retrabalho, 0 indicador foi considerado relevante, no entanto, foi apontada a necessidade de diferenciação dessas horas em motivos internos (qualidade do projeto, necessidade de novos treinamentos, não conformidades, dentre outros) e externos (como, por exemplo, pedidos de alteração e mudança de escopo pelo contratante).

Já a categoria qualidade, incorporou "melhoria nos processos produtivos" como estava disposto anteriormente no SMD existente da empresa. Desse modo, tal indicador saiu da categoria resultado, a qual adotou dois indicadores novos, "taxa de propostas financeiras fechadas" e "expansão do número de clientes", sendo que este último foi escolhido entre outras possibilidades, como expansão em áreas de atuação. Assim, a categoria resultados incorporou somente indicadores que influenciam diretamente nas finanças.

Por fim, a categoria inovação e sustentabilidade, com poucas modificações. $O$ indicador de número de inovações por projetos, mantido na proposta do SMD inicial, foi retirado, pois foi apontado pela diretoria da empresa como um indicador falho, por ser subjetivo e de difícil mensuração no âmbito do projeto. Em seu lugar, outro indicador foi adotado a partir da referência de Plentz (2014), o de investimento em P\&D por receita, que permite avaliar o quanto está sendo despendido em inovação e o quanto os investimentos estão apresentando resultados.

\section{Conclusões}

O interesse do presente estudo surgiu a partir da observação empírica sobre a carência de pesquisas destinadas aos sistemas de medição de desempenho em empresas de projeto no âmbito da construção civil, e de falhas de gestão nessas empresas, das quais se destaca a ausência de ferramentas de controle, ou correto uso das mesmas para mensuração do desempenho.

O principal objetivo estabelecido foi propor um SMD para uma empresa de projetos de arquitetura, com o intuito de contribuir para avanços no gerenciamento de empresas do setor da construção, assim como elevar o nível de sucesso de projetos foi atendido.

Para tanto, definiu-se os critérios para a seleção de uma empresa para realização do estudo caso, dos quais, possuir um SGQ consolidado foi essencial, por sua evidente contribuição na proposição do SMD, tanto no que se refere aos documentos e base de dados existentes na empresa estudada, quanto em relação à consciência da direção da empresa na busca por melhorias na gestão. 
Após aplicação do questionário e entrevista, os dados coletados juntamente com outros documentos fornecidos foram a base para as análises e proposição do sistema. Na etapa de processamento dos dados, constatou-se que apesar de monitorar o atingimento dos objetivos estratégicos, é fundamental avaliar as atividades que contribuem para os resultados esperados. Também foi identificada como uma das etapas imprescindíveis para a manutenção do SMD, a revisão, uma vez que o sistema não é estático e precisa acompanhar as mudanças ocorridas na organização.

No sistema inicial, dos 12 indicadores do SMD atual da empresa, 9 foram mantidos sem alterações, 1 foi modificado, e 2 foram integrados em um único, com o objetivo de não se distanciar das metas e interesses da empresa estudada, pois uma das principais recomendações encontrada na literatura é o alinhamento entre indicadores e objetivos estratégicos da organização. Ao mesmo tempo, foram consideradas premissas básicas para que o sistema fosse capaz de atender a outras empresas do setor.

Após a conclusão da proposta, buscou-se incorporar a visão da empresa para um possível aprimoramento do SMD, o que resultou no refinamento dos indicadores, que consequentemente estabelecem uma proximidade maior com a realidade de uma empresa de projetos de pequeno porte.

Quanto aos checklists mencionados no decorrer do texto, entende-se que cada empresa deve criar os seus próprios de acordo com os métodos e os processos que possui. No caso da empresa analisada, os checklists já existem e são monitorados de forma consistente. Como sugestão para as empresas que não possuem, há exemplos norteadores disponibilizados na literatura.

Como limitação, destaca-se o fato do estudo ser realizado em apenas uma empresa, e a limitação de pesquisas para a revisão bibliográfica com estudos de caso que abordam SMD na prática de empresas de arquitetura. Dessa forma, recomenda-se para próximas pesquisas a realização de um número maior de estudos de caso, possibilitando a comparação entre os sistemas e a identificação das necessidades e limitações comuns.

Por fim, após a construção final do sistema, acredita-se que os objetivos propostos inicialmente foram cumpridos, pois o SMD pode ser considerado um modelo para aprimoramento ou implementação, e sua estrutura permite adaptações de acordo a necessidade de cada empresa. E ainda contribui para futuras pesquisas nesse âmbito.

\section{Agradecimentos}

À empresa Lins Galvão que se dispôs prontamente a contribuir com este estudo. Agradecemos pela atenção e cordialidade por parte da direção, e pela disponibilização de informações e documentos que foram essenciais para a realização da proposta.

\section{Referências bibliográficas}

ASSOCIAÇÃO BRASILEIRA DE NORMAS TÉCNICAS. NBR 15575: Edificações habitacionais Desempenho. Rio de Janeiro, 2013.

ASSOCIAÇÃO BRASILEIRA DE NORMAS TÉCNICAS. NBR ISO 9001: Sistemas de gestão da qualidade - Requisitos. Rio de Janeiro, 2008.

ALI, H. ABD E. M.; AL-SULAIHI, I. A.; AL-GAHTANI, K. S. Indicators for measuring performance of building construction companies in Kingdom of Saudi Arabia. Journal of King Saud University Engineering Sciences, v. 25, n. 2, p. 125-134, 2013.

BERNARDI, D. C. O uso de indicadores de desempenho no processo de monitoramento e controle de projetos. Monografia (Especialização em Gestão de TI) - Universidade Federal de Santa Maria, Frederico Westphalen, RS, 2013. 
BEUREN, I. M.; FLORIANI, R.; HEIN, N. Indicadores de inovação nas empresas de construção civil de Santa Catarina que aderiram ao Programa Brasileiro de Qualidade e Produtividade no Habitat (PBQP-H). Perspectivas em Gestão \& Conhecimento, v. 4, n. 1, p. 161-178, jan./jun. 2014.

BINGOL B. N.; POLAT G. Measuring Managerial Capability of Subcontractors Using a KPI Model. Creative Construction Conference, v. 196, p. 68-75, jun. 2017.

CÂNDIDO, L. F.; LIMA, S. H. de O.; BARROS NETO, J. de P. Análise de sistemas de medição de desempenho na indústria da construção. Ambiente Construído, Porto Alegre, v. 16, n. 2, p. 189-208, abr./jun. 2016.

CELLI, R. C. Indicador custo de não conformidade como instrumento de tomada de decisão nas micro e pequenas empresas título: Um Estudo de Caso em uma MPE do Setor Metalúrgico. Dissertação (Mestrado em Administração) - Faculdade Campo Limpo Paulista. Campo Limpo Paulista, SP, 2014.

COSTA, D. B. Diretrizes para concepção, implementação e uso de sistemas de indicadores de desempenho para empresas da construção civil. Dissertação (Mestrado em Engenharia Civil) Universidade Federal do Rio Grande do Sul. Porto Alegre, 2003.

DENG F.; SMYTH H. J.; ANVUUR A. M. A critical review of PMS in construction: Towards a research agenda. Annual ARCOM Conference, p. 807-816, sept. 2012.

FERNANDES, D. R. Uma contribuição sobre a construção de indicadores e sua importância para a gestão empresarial. Revista FAE, Curitiba, v.7, n.1, p. 1-18, jan./jun. 2004.

FRANCO-SANTOS, M.; LUCIANETTI, L.; BOURNE, M. Contemporary Performance Measurement Systems: a review of their consequences and a framework for research. Management Accounting Research, v. 23, n. 2, p. 79-119, 2012.

GARENGO, P.; BIAZZO, S.; BITITCI, U. S. Performance measurement systems in SMEs: a review for a research agenda. International Journal of Management Reviews, v.7, n.1, p. 25-47, mar. 2005.

HORSTMAN, A. Performance Indicators in the Best Value Approach: Proposed process for developing and using performance indicators for infrastructural projects approached with Best Value. Master programme (Civil Engineering \& Management) - University of Twente. Enschede, sept. 2013.

JIN, Z.; DENG F.; LI, H.; SKITMORE M. Practical Framework for Measuring Performance of International Construction Firms. Journal of construction engineering and management, v. 139, p.1154-1167, sept. 2013.

KASSEM, M.; AMORIM, S. R. L. BIM - Building Information Modeling no Brasil e na União Europeia. Brasília: Ministério do Planejamento, Orçamento e Gestão, 2015. Disponível em: $<$ http://sectordialogues.org/ sites/default/files/acoes/documentos/bim. pdf>. Acesso em: 03 maio 2018.

MADRIGAL, B. E. B. Sistema de Indicadores de Gestão para Monitorização Estratégica. Dissertação (Mestrado em Contabilidade e Finanças) - Instituto Politécnico de Bragança. Bragança, 2012.

MARTINS, V. W. B; NEVES, R. M. das; MACÊDO, A. N. Análise do desenvolvimento de competências gerenciais na construção civil através do modelo da Aprendizagem Baseada em Problemas adaptado ao contexto organizacional. Ambiente Construído, Porto Alegre, v. 14, n. 1, p. 155-175, jan./mar. 2014. 
MELLO, L. C. B. de B.; AMORIM, S. R. L. de; BANDEIRA, R. A. de M. Um sistema de indicadores para comparação entre organizações: 0 caso das pequenas e médias empresas de construção civil. Gestão \& Produção, v. 15, n. 2, p. 261-274, maio/ago. 2008.

MITCHELL, R. K.; AGLE, B. R.; WOOD, D. J. Toward a theory of stakeholder identification and salience: defining the principle of who and what really counts. Academy of Management Review, v. 22 , n. 4 , p. $853-886,1997$.

MORAES, A. F. de S.; PICCHI, F.; GRANJA, A. D. Variáveis e índices geométricos de projeto arquitetônico relacionados ao custo de empreendimentos residenciais. XVI Encontro Nacional de Tecnologia do Ambiente Construído, 16., 2016, São Paulo. Anais... Porto Alegre: ANTAC, 2016.

NEELY, A.; GREGORY M.; PLATTS K. Performance measurement system design: A literature review and research agenda. International Journal of Operation \& Production Management, Cambridge, v. 25, p. 1128-1263, 2005.

NÓBREGA JÚNIOR, C. L.; MELHADO, S. B. Coordenador de projetos de edificações: estudo e proposta para perfil, atividades e autonomia. São Paulo: EPUSP, 2013. 26 p. (Boletim Técnico da Escola Politécnica da USP, Departamento de Engenharia de Construção Civil, BT/PCC/579).

ORIHUELA, P.; PACHECO S.; AGUILAR, R.; ORIHUELA, J. Propuesta de Indicadores de Resultado para Proyectos de Edificación. VII Elagec - II SelN²co, Nuevas Tendencias em la Construcción Sostenible, 2016.

PEREIRA, A. G. de L. Análise de não conformidades de projetos na etapa de construção de edifícios. Monografia (Especialização em Gestão de Projetos na Construção) - Escola Politécnica da Universidade de São Paulo. São Paulo, 2017.

PLENTZ, N. D. Proposição de um sistema de indicadores de inovação, competitividade e design voltado para empresas desenvolvedoras de produtos. Dissertação (Mestrado em Design) Universidade Federal do Rio Grande do Sul. Porto Alegre, 2014.

RODRIGUES, A; CESÁRIO F.; CASTANHEIRA F.; CORREIO M. C. Gestão de desempenho: validação de um instrumento de medida. Revista de Ciências da Administração, v. 19, n. 47, p. 5774, abril 2017.

RONCON A.; OLIVEIRA, M. L.; BELTRAME, I. Processos gerenciais de gestão de pessoas em empresas do setor da construção civil. Revista de Carreira e Pessoas, p. 114-128, 2015.

ROZADOS, H. B. F. Uso de indicadores na gestão de recursos de informação. Revista Digital de Biblioteconomia e Ciência da Informação, v. 3, n. 1, p. 60-76, jul./dez. 2005.

SILVA D. A. dos R.; FLEURY A. L.; CARVALHO M. M. de. Sucesso no gerenciamento de projetos e projetos conforme a visão de diferentes stakeholders: uma pesquisa ação sobre o novo sistema de gerenciamento de relacionamento com o cliente. XXXV Encontro Nacional de Engenharia de Produção. Perspectivas Globais para a Engenharia de Produção. Fortaleza, outubro 2015.

SONNENTAG, S.; FRESE, M. Performance Concepts and Performance Theory. Psychological Management of Individual Performance, 2002.

SOUZA, D. da M. O. Sistemas de medição de desempenho para projetos de PD\&I no setor de petróleo e gás natural. Tese (Doutorado em Ciência e Engenharia de Petróleo) - Universidade Federal do Rio Grande do Norte. Natal, 2014. 